\title{
Comparative study between Ultrasonography and Optical Coherence Tomography in interventional cardiology
}

\author{
Félix Fanjul-Vélez*a , José María de la Torre-Hernández ${ }^{\mathrm{b}}$, Noé Ortega-Quijano ${ }^{\mathrm{a}}$, José Javier Zueco- \\ Gil $^{\mathrm{b}}$, José Luis Arce-Diego ${ }^{\mathrm{a}}$ \\ ${ }^{a}$ Applied Optical Techniques Group, TEISA Department, University of Cantabria, Av. de los \\ Castros s/n, 39005 Santander, Spain \\ ${ }^{\mathrm{b}}$ Interventional Cardiology Department, Marqués de Valdecilla University Hospital, Av. Valdecilla \\ s/n, 39008 Santander, Spain
}

\begin{abstract}
In this work, we present clinical images of IVUS and OCT in the evaluation of pharmacological stent endothelization. These preliminary imaging results are analyzed and compared in order to determine the ability of these technologies to visualize relevant intravascular features of interest in interventional cardiology. The results enable to compare the performance of both techniques and to evaluate their potential for clinical purposes.
\end{abstract}

Keywords: Optical Coherence Tomography, Intravascular Ultrasonography, interventional cardiology, drug-eluting stent, pharmacological stent

\section{INTRODUCTION}

Cardiovascular diseases are the leading cause of death worldwide. In developed countries, heart diseases provoke more than a fourth of the total deaths, thus having more impact than malignant neoplasms [1]. The main cause of acute myocardial infarction is the rupture of the vulnerable plaque followed by vessel occlusion and subsequent thrombosis. In particular, the most dangerous plaques, i.e. those who are more likely to suffer a rupture, are those with a thin and structurally weak fibrous cap [2]. These thin-cap fibroatheromas (TCFAs) are characterized by a critical cap thickness of about $65 \mu \mathrm{m}$ [3], a large lipidic fold, and activated macrophages.

A deep study of the factors involved in plaque rupture is needed in order to perform an early identification of high-risk lesions and prevent diseases by an adequate therapy. In order to do this, a number of intravascular imaging technologies are available. Among them, those with the greater potential to adequately study arterial plaques are Intravascular Ultrasound (IVUS) and Optical Coherence Tomography (OCT). Their usefulness in interventional cardiology arises from their resolution and their ability to visualize intravascular features such as lipids and calcified tissues, for example. In this work, the features shown by both imaging modalities will be analyzed, highlighting the great potential of OCT for intravascular applications.

As well as TCFA study, the monitoring of stents after coronary angioplasty is very important in cardiology. Both IVUS and OCT can be used to evaluate the adequate positioning of the stent within the artery lumen, which is essential to maintain the vessel opened. Stent endothelization is another key factor involved in the succesfulness of the process, as long as it decisively contributes to the stent implantation and fixing. As a way to improve stent angioplasty, pharmacological stents are beginning to be used as a way to avoid the growth of scar tissue around the stent and subsequent vessel occlusion. The endothelization process in drug-eluting stents is of particular importance. IVUS and OCT play a key role in the assessment of this feature.

In this work, IVUS and OCT are analyzed, applying them to perform preliminary imaging of arteries with stents for cardiological applications. In section 2, the intravascular features involved in plaque analysis are presented. The different imaging modalities available for their study are mentioned, emphasizing the potential of IVUS and OCT over the rest of them. In section 3, the processes involved in coronary angioplasty are briefly revised, with special attention to stent angioplasty. Finally, section 4 shows some clinical results of IVUS and OCT in the evaluation of pharmacological stent endothelization. These results enable to compare the performance of both techniques and to evaluate their potential for clinical purposes.

Optical Coherence Tomography and Coherence Techniques IV, edited by Peter E. Andersen, Brett E. Bouma, Proc. of SPIE-OSA Biomedical Optics, SPIE Vol. 7372, 73722D

(C) 2009 SPIE-OSA · CCC code: 1605-7422/09/\$18 - doi: 10.1117/12.831842 


\section{INTRAVASCULAR IMAGING MODALITIES FOR PLAQUE ANALYSIS}

Atherosclerosis consists on the growth of a plaque on the inner walls of the arteries, under a fibrous cap, which results in a narrowing of the available vessel diameter for blood circulation. If atherosclerosis appears in the coronary arteries, then it receives the name of Coronary Artery Disease (CAD). Among all types of coronary artery plaques, the most dangerous ones are vulnerable plaques, i.e. those who are more prone to suffer a rupture. If plaque rupture is produced, then the plaque fragment is transported by the oxygen-rich blood flow to the heart, and when the artery diameter decreases it can cause vessel occlusion. Thrombosis obstructs blood flow and then a loss of blood supply is produced, causing infarction.

Therefore, vulnerable plaque rupture is the main cause of acute myocardial infarction. The main characteristic defining a vulnerable plaque is a thin and structurally weak fibrous cap [2]. Thus, they are defined as TCFAs. The critical thickness of the fibrous layer in order to consider that it is a high-risk plaque is $65 \mu \mathrm{m}$ [3]. As well as this, there are other important factors to take into account in plaque characterization and study, like the presence of a large lipid fold and activated macrophages near the fibrous cap [3]. Both the thin fibrous layer and the large and highly lipidic fold can be observed in the histological image shown in Figure 1.

In order to localize plaques in vivo and to determine whether it is vulnerable or not, intravascular imaging techniques are needed. Several imaging techniques have been developed in the last decade in order to enhance the vulnerable-plaque detection. The most relevant are coronary angiography, intravascular MRI, angioscopy, intravascular ultrasound and, recently, optical coherence tomography [4]. The detection of fibroatheroma thin-cap is a very demanding operation that requires a very high resolution, as long as it is necessary to determine the critical cap thickness with a reasonable degree of accuracy. Therefore, the most powerful techniques for TCFA detection are intravascular ultrasound and optical coherence tomography. However, they show great differences in terms of resolution, soft-tissue contrast, penetration depth, and distinguishable coronary features such as lipid cores, calcium and thrombus.

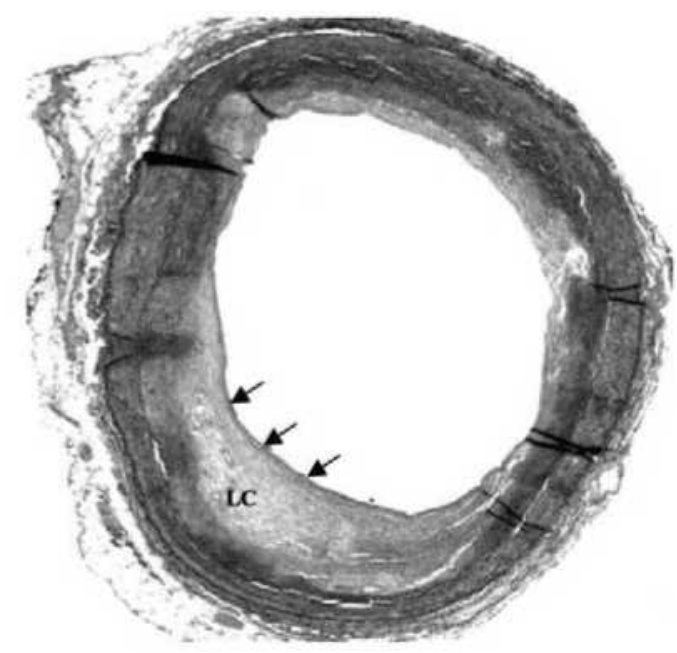

Fig. 1. Histological image of a vulnerable coronary plaque. The thin fibrous cap and the highly lipidic core can be observed (they are marked with arrows and 'LC' respectively) [4].

Intravascular Ultrasound (IVUS) is a well-established method to perform tomographic imaging of the coronary arteries. However, its resolution of around $100 \mu \mathrm{m}$ is very limited to accurately determine the thickness of the fibrous cap [4]. Optical Coherence Tomography (OCT) is a very powerful technique with a wide range of applications [5]. It has shown to be a robust technique to perform high-resolution imaging for plaque characterization [6]. Its capacity to visualize the fibrous cap and to quantify the macrophages with a 10 times better resolution than IVUS makes OCT a powerful tool to perform evaluation of different plaque types. As well as TCFA detection, both IVUS and OCT can be applied to other interventional cardiology applications that also require high resolution [7]. The most evident examples are the determination of stent malapposition and the evaluation of stent endothelization, in particular after pharmacological stent implantation, as will be explained in next section. 


\section{STENT CORONARY ANGIOPLASTY}

Coronary angioplasty, also known as percutaneous coronary intervention (PCI) or percutaneous transluminal coronary angioplasty (PTCA), is a medical interventional procedure to open an atherosclerotic artery by eliminating the occlusive agent, usually an arterial plaque. The result is a broadening of the lumen and the restoration of normal blood flow to the heart. Angioplasty is widely used then the arterial occlusion is not very severe, so that the catheter can reach the blocking plaque. In the case of severe occlusion, multiple arteries blocking, or even heart failure, coronary artery bypass grafting (CABG) has to be used. CABG involves open-heart surgery, so coronary angioplasty tends to be used as far as possible. In comparison with bypass grafting, angioplasty does not require surgery, so it results in a shorter recovery time, and it entails fewer risks during and immediately after the procedure. As well as this, angioplasty is also very valuable during a heart attack, as long as it enables a quick arterial opening, which is a key factor in minimizing the damage to the heart as a result of interrumpted blood supply.

The simplest coronary angioplasty is performed by means of a balloon catheter. Firstly, an angiogram has to be made in order to locate the CAD. The catheter is introduced through the groin or the arm. Once it has been threaded to the coronary arteries, a contrast dye is injected before taking the radiological image. Having located the blockage with the aid of this X-ray picture, the catheter with the deflated balloon is situated next to the plaque, and then it is blown up to compress the plaque against the inner artery wall. After deflating the balloon and puting out the catheter, normal blood flow is restored in the previously occluded region.

One of the main disadvantages of balloon coronary angioplasty is that a narrowing of the artery over time is sometimes observed. In order to overcome this, intravascular stents are used. A stent is a cylindrical metalic mesh that can be attached to the balloon catheter in the closed state, and when the balloon is inflated the stent expands, as shown in Figure 2.
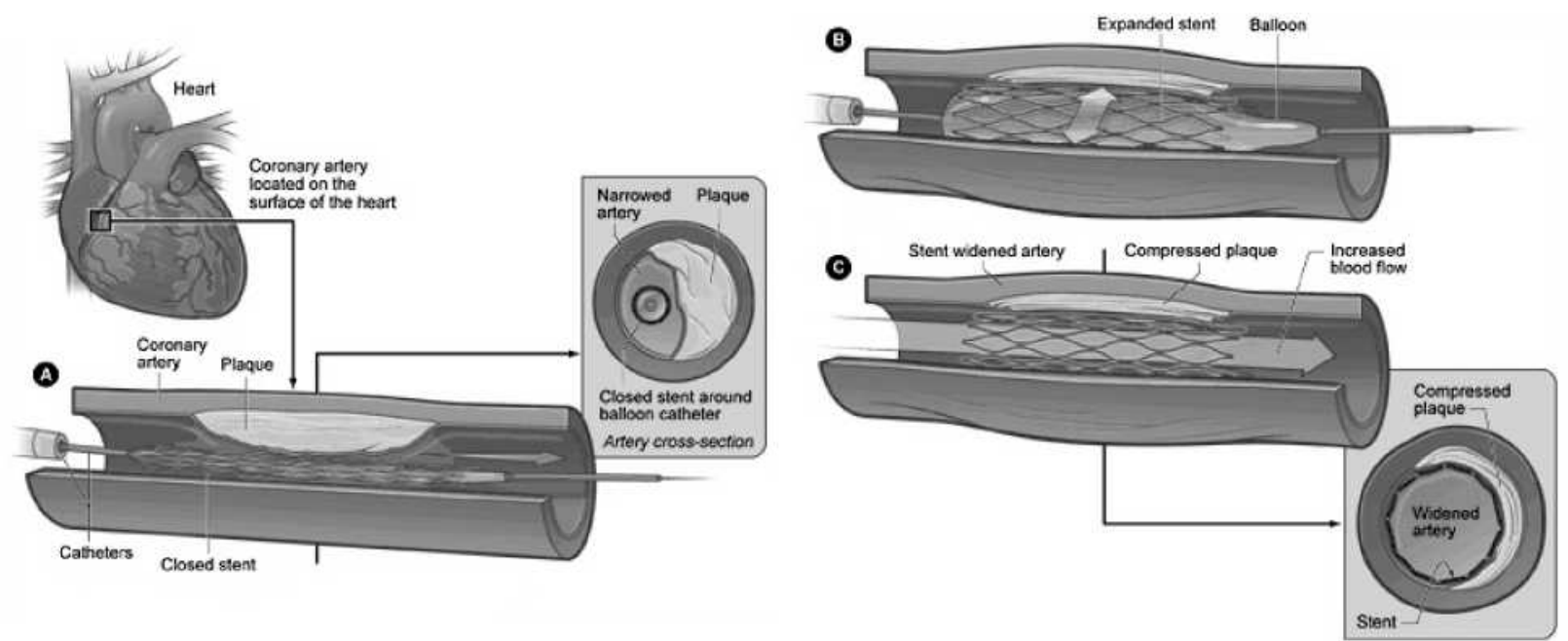

Fig. 2. Coronary stent placement. A) Insertion of a balloon catheter with a closed stent attached to it (inset shows an arterial cross-section where the catheter position and the plaque can be observed). B) Balloon inflation causes stent expansion, and subsequently plaque compression and artery widening. C) Once the catheter with the balloon has been retired, the stent maintains the structure of the arterial lumen, as it is shown in the inset [National Heart Lung and Blood Institute, U.S. Department of Health and Human Services].

Stents are designed to maintain its shape over the time, so that the artery remains widened. Therefore, the risk of artery renarrowing with stent angioplasty is low. Nevertheless, the formation of scar tissue around the stent can cause occlusion. In order to avoid this, it is possible to make use of an improved type of stents: drug-eluting stengs, also named pharmacological stents or medicine-coated stents. These stents release drugs to the artery in a continuous and maintained way, helping to avoid the growth of new tissue around them and then preventing blocking. Another important factors in the successfulness of stent angioplasty is the appropriate stent positioning within the artery and the endothelization process that helps to fix it to the inner wall of the vessel. 


\section{COMPARATIVE STUDY OF IVUS AND OCT IN PHARMACOLOGICAL STENT ENDOTHELIZATION ASSESSMENT}

Pharmacological stent endothelization can be estimated by means of several imaging techniques. Perhaps the most relevant ones are those highlighted in section 2, intravascular ultrasound and optical coherence tomography. The first one is widely used in intravascular imaging, while the latter is being adopted in hospitals around the world in recent years.

In this work, a comparison of both techniques in interventional cardiology applications is shown. In particular, we present the preliminary results of neointimal and thrombotic tissues formation imaging around drug-eluting stents. Two commercially-available equipments have been employed to obtain intravascular tomographic images of coronary arteries. The procedure was performed by specialized practitioners of the Interventional Cardiology Department of Marqués de Valdecilla University Hospital (Santander, Spain), following the standardized clinical procedures. The IVUS equipment employed is a solid-state 64-crystals phased-array $20 \mathrm{MHz}$ IVUS scanner (Volcano Therapeutics Inc., California) [8]. It shows a depth resolution of $150 \mu \mathrm{m}$, and a penetration depth of a few millimeters. The OCT equipment is a recently developed time domain implementation (LightLab Imaging, Massachussets) [9]. It makes use of a SLED with a central wavelength of $1300 \mathrm{~nm}$ and a FWHM of $50 \mathrm{~nm}$. It achieves a depth resolution of 10-15 $\mu \mathrm{m}$ with an optical sensitivity of $90 \mathrm{~dB}$. The penetration depth varies with the tissue components but an approximate value for all cases is 1 $\mathrm{mm}$. The operation is performed at $1 \mathrm{~mm} / \mathrm{seg}$, taking 20 frames per second at 240 radial lines per frame.

a)

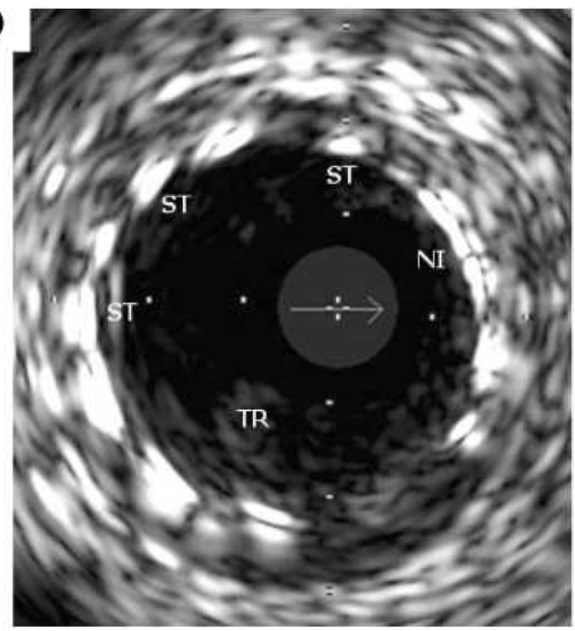

b)

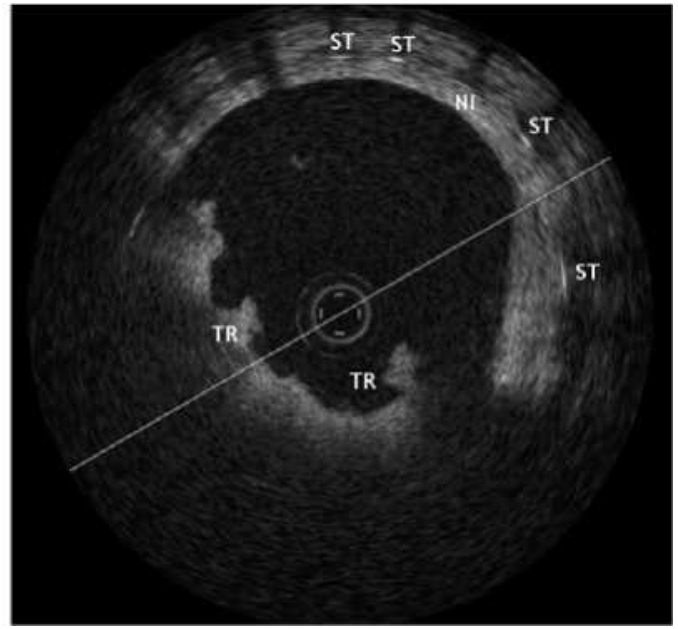

Fig. 3. Tomographic imaging of a drug-eluting stent (ST) endothelization with neointimal (NI) tissue and thrombus (TR) formation. A) IVUS image. B) OCT image.

Figure 3 shows a comparison between an IVUS and an OCT image. It has been taken to monitor the evolution of a drugeluting or pharmacological stent. The presence of scar tissue and thrombus formation after treatment with this type of stents is a common issue [10]. The threads of the stent are clearly seen in both images, corresponding to the small regions with high reflectivity noted by ' $\mathrm{ST}$ ' in both images (only a few have been noted). The main difference between both techniques is their capacity to distinguish between neointimal tissue ('NI') and thrombus ('TR'). IVUS does not present enough soft tissue contrast to accurately establish a differential diagnostics between them. On the contrary, it can be observed that the image obtained by OCT clearly differentiates both tissues. Moreover, the thickness of the neointimal tissue surrounding the stent can be determined. It should also be noted that the stent thickness is about $100 \mu \mathrm{m}$. The radial dark lines observed in the OCT image are no other than the signal shadows caused by the metalic threads of the stent, as long as they show a high reflectance for optical wavelenghts. This effect is not observed in IVUS image due to the mechanical characteristics of the sound waves.

The incipient formation of neointimal tissue on a drug-eluting stent can be observed in Figure 4. It constitutes a key factor to ensure a successful stent implantation. Lack of endothelization may result in the growth of potentially dangerous thrombotic tissue [11]. The IVUS image shows a poor resolution that does not enable the practitioner to observe any neointimal tissue formation. However, OCT shows a thin neointimal layer that has appeared on a stent thread (at right). Its thickness is approximately the same as that of the stent, i.e., $100 \mu \mathrm{m}$. The other stent thread noted in the image does not seem to have been covered by neointimal tissue. 
a)
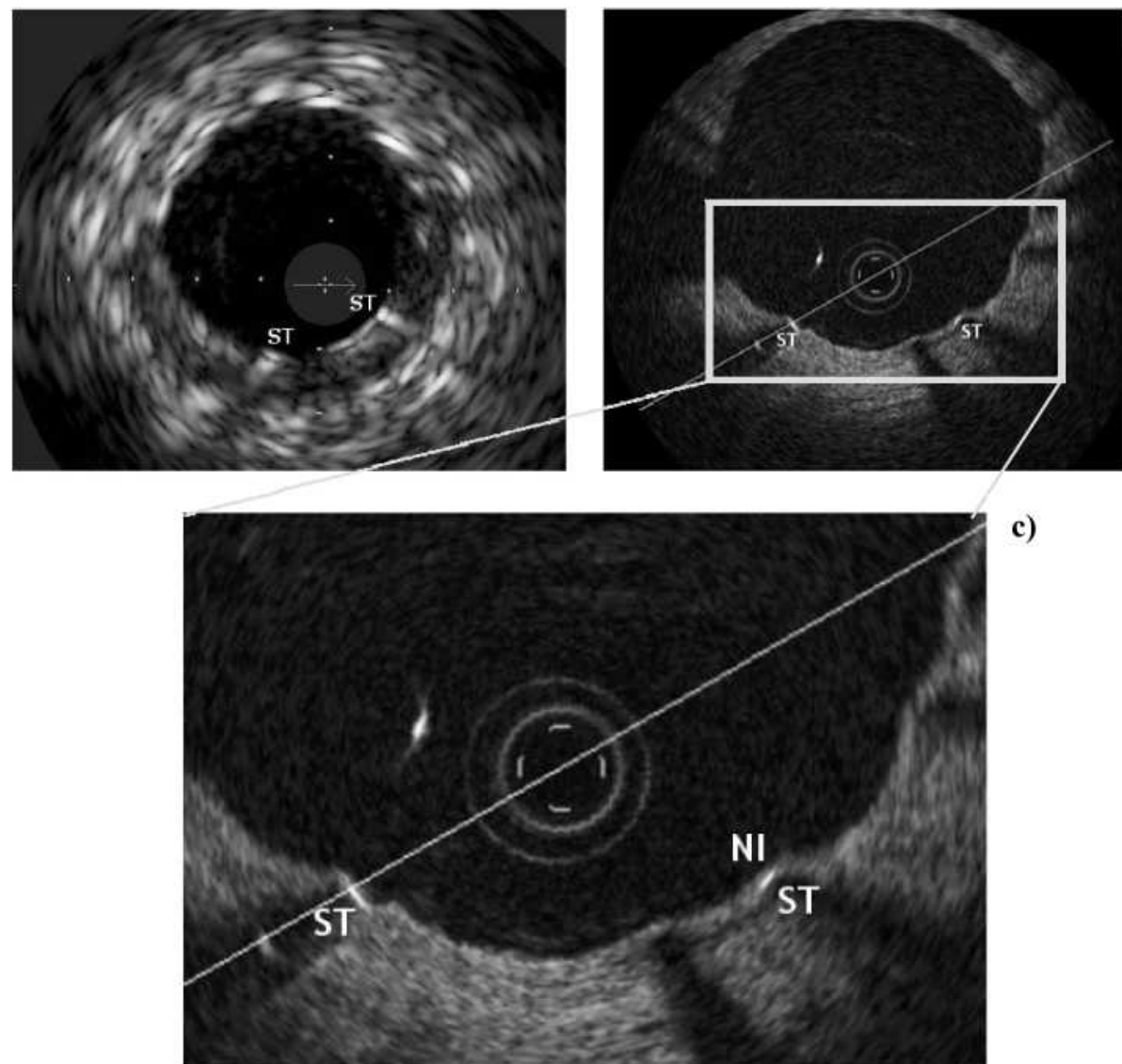

Fig. 4. Tomographic imaging of a drug-eluting stent (ST) incipient endothelization with neointimal (NI) tissue recently formed. A) IVUS image. B) OCT image. C) OCT image (detail)

\section{CONCLUSIONS}

These preliminary images of pharmacological stents endothelization highlight the limitations of IVUS, mainly due to its limited resolution and its poor soft tissue contrast. Although its penetration depth is very high, its poor resolution does not enable to accurately perform measurements of coronary arteries features. As well as that, thrombotic and neointimal tissues are not clearly differentiated in IVUS images. OCT has shown to be able to distinguish between thrombus and neointima in a very robust way, and the tomographic images present a very sharp delineation of such structures. Additionally, its very high resolution enables to evaluate neointimal tissue growth at a thickness unaffordable for IVUS. These results demonstrate the high potential of OCT for its application in intravascular cardiology.

\section{ACKNOWLEDGMENTS}

This work has been carried out partially under the project TEC2006-06548/TCM of the Spanish Ministry of Education and Science.

\section{REFERENCES}

[1] American Cancer Society, "Global Cancer Facts \& Figures” (2007). 
Brezinski, M. E., Tearney, G. J., Bouma, B. E., Izatt, J. A., Hee, M. R., Swanson, E. A., Southern, J. F. and Fujimoto, J. G., "Optical Coherence Tomography for optical biopsy. Properties and demonstration of vascular pathology", Circulation 93(6) March 75, 1206-13 (1996).

[3] Xu, C., Schmitt, J. M., Carlier, S. G., Virmani, R., "Characterization of atherosclerosis plaques by measuring both backscattering and attenuation coefficients in Optical Coherence Tomography", Journal of Biomedical Optics 13(3), 034003-1-8 (2008).

MacNeill, B. D., Lowe, H. C., Takano, M., Fuster, V. and Jang, I.-K., "Intravascular modalities for detection of vulnerable plaque: current status", Arterioscler. Thromb. Vasc. Biol. 23, 1333-1342 (2003).

[5] D. Pereda-Cubián, M. Todorovic, J. L. Arce-Diego, L. Wang, "Evaluation of the magneto-optical effect in biological tissue models using optical coherence tomography", Journal of Biomedical Optics 12(6), 060502 (2007).

[6] Low, A. F., Tearney, G. J., Bouma, B. E. and Jang, I.-K., "Technology insight: Optical Coherence Tomographycurrent status and future development", Nature Clinical Practice Cardiovascular Medicine 3(3), 154-62 (2006).

[7] Pinto, T. L. and Waksman, R., "Clinical applications of Optical Coherence Tomography", Journal of Interventional Cardiology 19(6), 566-73 (2006).

[8] Volcano Therapeutics Inc., "Ivus Imaging", http://www.volcanocorp.com/products/ivus-imaging/index.asp

[9] LightLab Imaging, "OCT Technology", http://www.lightlabimaging.com/intl/OCTtech/intro.htm

[10] J. M. de la Torre-Hernández, F. Alfonso, F. Hernández, J. Elizaga, M. Sanmartin, E. Pinar et al., "Drug-eluting stent thrombosis: results from the multi-center Spanish Registry ESTROFA", J. Am. Coll. Cardiol. 51, 986-90 (2008).

[11] Jakabcin, J., Bystron, M., Spacek, R., Veselka, J., Kvasnak, M., Kala, P., Malý, J., Cervinka, P., "The lack of endothelization after drug-eluting stent implantation as a cause of fatal late stent thrombosis", J. Thromb. Thrombolysis 26(2), 154-8 (2008). 